\title{
Treatment guidelines for nosocomial pneumonia: Agreeing to disagree
}

$\mathrm{C}$ ONSENSUS CONFERENCES TO GENERATE TREATMENT guidelines for specific infectious diseases or syndromes seem to be something of a growth industry. In this issue of The Canadian Journal of Infectious Diseases we are presented with another, this one on the initial antimicrobial treatment of nosocomial pneumonia (pages 317-321). To begin to sort out how to make optimal use of these particular guidelines, we should first consider what requirements underly the development of a meaningful consensus of this kind.

First, we need a diagnosis clearly enough defined that consensus seekers can agree to it and guideline users can recognize it. If the case definition is vague or subjective, even the best treatment guidelines cannot ensure uniform standards of care. Second, it is helpful if the usual microbial etiology of the diagnosis in question is known and is reasonably uniform in different places and at different times. Third, there should be good comparative data regarding efficacy and cost for potential treatment regimens, so that recommendations can be both specific and justifiable. Without this, while guidelines may, at best, represent the distilled wisdom of the shared experience of experts, they risk being merely codified bias. When these requirements are met, guidelines can make an important contribution to educating physicians about appropriate management of infectious diseases. The Canadian Treatment Guidelines for Sexually Transmitted Diseases is an example of a consensus document which is both useful and authoritative, in which the diagnostic entities are well-defined, the responsible pathogens are known and generally consistent in kind and antimicrobial susceptibility, and which has good comparative data of treatment efficacy and cost benefit to support specific antimicrobial recommendations (1).

An examination of the recommended antimicrobial regimens for nosocomial pneumonia suggests that the consensus of conference participants was to agree to disagree. This is likely because they were trying to reach a consensus without any of the requirements noted above being met. First, there is considerable debate about diagnostic criteria for nosocomial pneumonia. In intubated patients, for example, some authors referenced in the consensus statement argue that more than half of patients meeting conventional clinical criteria for pneumonia do not have lung infection $(2,3)$. I presume these disagreements explain why the consensus document neither provides a case definition of nosocomial pneumonia, nor addresses the clinical scenario of doubtful diagnosis. Second, while some generalizations regarding the microbial etiology of nosocomial pneumonia are possible, local endemic nosocomial pathogens are highly variable in type and antimicrobial susceptibility. Finally, few comparative treatment data are available to support specific treatment recommendations for nosocomial pneumonia.

The difficulties faced by the conference participants are exemplified by the treatment recommendations for mild to moderate nosocomial pneumonia shown in the tables ( 1 and 2) in the treatment guidelines. For a patient with recent upper abdominal surgery who develops a mild to moderate postoperative pneumonia, the listed treatment options range from oral cotrimoxazole to intravenous cefotaxime with clindamycin. In every risk category listed in the tables multiple treatment options, covering the spectrum from older, less expensive drugs to the most recent and expensive agents, are suggested. No guidance is offered to assist in selecting among them.

The fundamental problem is that, for a disease like nosocomial pneumonia, it is not possible to make a specific recommendation that will be valid for all hospi- 
tals at all times. If a naïve physician selects cefazolin/gentamicin for pneumonia treatment in a hospital with endemic resistant Gram-negatives, he or she will be guilty of under-treatment. On the other hand, if imipenem/cilastatin with an aminoglycoside are used as empirical therapy for all patients in an intensive care unit without endemic resistant Gram-negatives, the cost effectiveness is questionable.

The emphasis in the consensus report on stating that the guidelines are meant as an aid to the physician, and "in all cases the individual physician dealing with pneumonia in a particular patient is ultimately in the best position to determine ideal therapy", seems to defeat the purpose of having guidelines. Guidelines are meant to educate physicians and to set a standard of care for efficacy and cost effectiveness. If they contain recommendations that, in a given hospital, may range from ineffective to expensive overkill, they cannot act as a useful guide to those who need them.

Perhaps the appropriate use of this document is as a foundation for the development of local consensus guidelines for individual hospitals or communities. Several principles which would permit more specific, limited, local recommendations are explicit in the report. These include: first, Staphylococcus aureus and facultative Gram-negative bacilli are important causes of nosocomial pneumonia; second, longer hospital stay and prior antimicrobial exposure are associated with increased risk of more resistant infections; third, endemic hospital acquired microorganisms vary greatly among hospitals in kind and in antimicrobial susceptibility; and fourth, severe infections associated with respiratory failure or shock carry a greater risk of death and mandate greater breadth and intensity of initial antimicrobial therapy. Consideration of these facts, together with specific local knowledge of the patient population, microbial ecology and antimicrobial susceptibility patterns, should allow the development of locally applicable recommendations with a narrower range of treatment options. This would be more effective in educating physicians, ensuring a basic standard of therapy and minimizing cost.

The difficulty in achieving consensus for the treatment of nosocomial pneumonia results directly from our limited knowledge about many aspects of this disease. Why is there such inter-institution and inter-unit variability in incidence of pneumonia? How can we control the acquisition of antimicrobial resistant pathogens by patients at risk? How do we diagnose pneumonia and distinguish it from atelectasis or chemical pneumonitis in these patients? How do we confirm the microbial etiology? Do we always need to? What is the role of anaerobic bacteria in nosocomial pneumonia? Are there clinically meaningful differences in efficacy among available antimicrobial regimens? Perhaps a future conference with more answers and fewer questions will achieve more consensus than is now possible. Even then, I suspect any consensus will be better addressed to principles underlying effective development of local guidelines rather than to provision of lists of antimicrobial treatment options.

\section{REFERENCES}

1. Health and Welfare Canada. Canadian guidelines for the prevention, diagnosis, management and treatment of sexually transmitted diseases in neonates, children, adolescents and adults. Can Commun Dis Rep 1992;18S1:1-213.

2. Fagon J-Y, Chastre J, Domart Y, et al. Nosocomial pneumonia in patients receiving continuous mechanical ventilation. Am Rev Respir Dis 1989;139:877-84.

3. Chastre J, Fagon J-Y, Gilbert DC. Diagnosis of nosocomial pneumonia in intensive care units. Eur J Clin Microbiol Infect Dis 1989;8:35-9. 


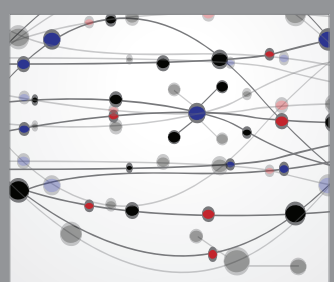

The Scientific World Journal
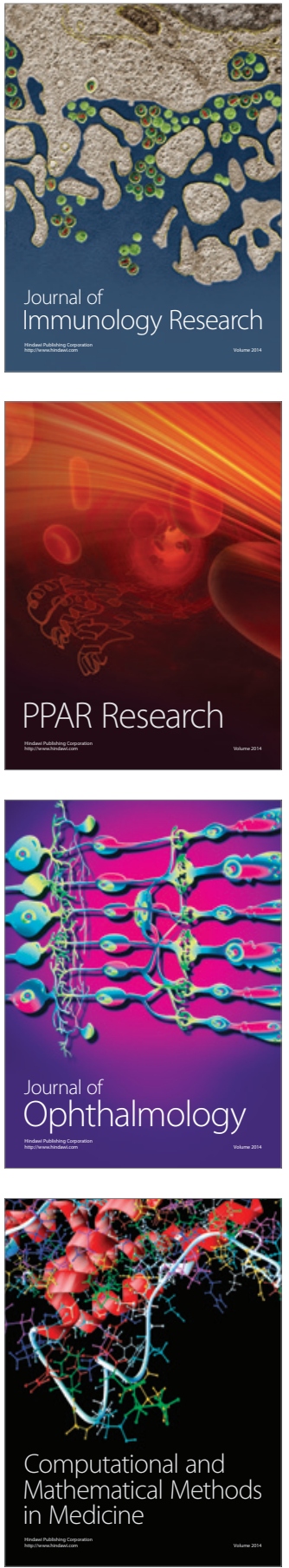

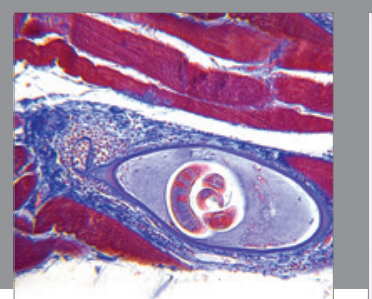

Gastroenterology Research and Practice

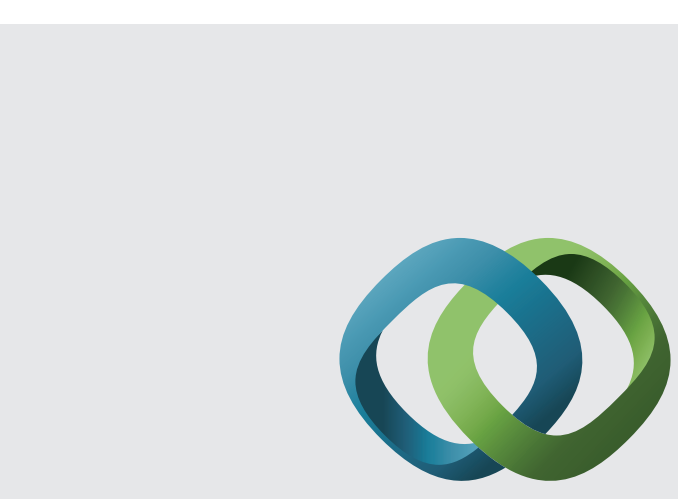

\section{Hindawi}

Submit your manuscripts at

http://www.hindawi.com
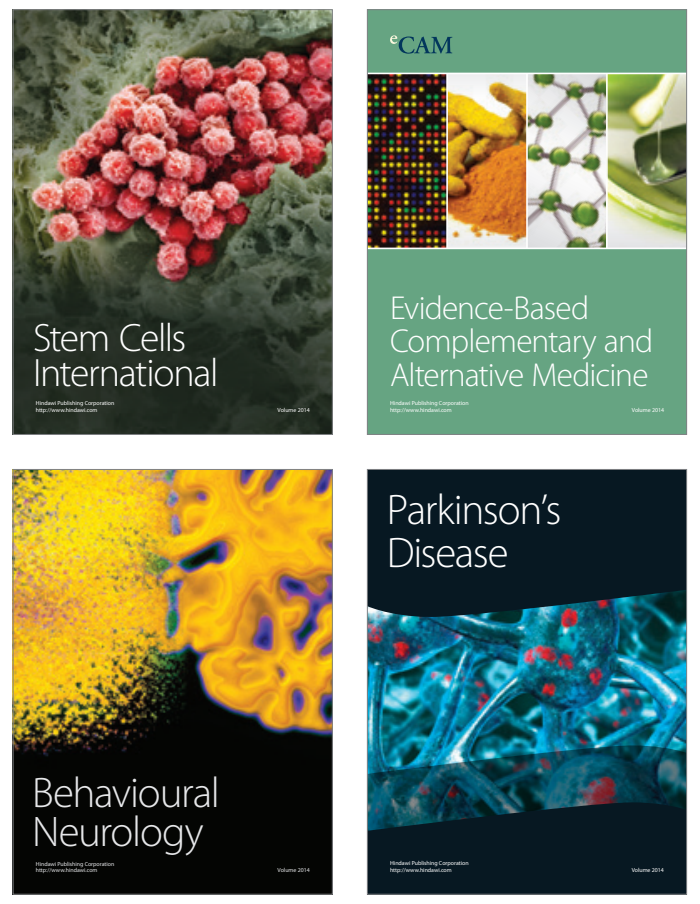
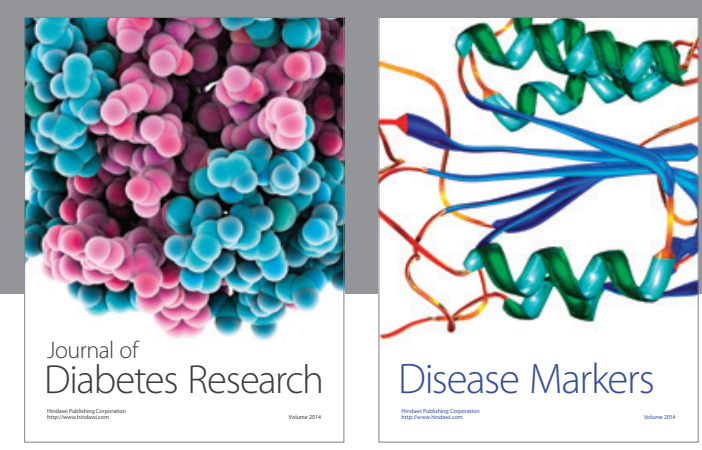

Disease Markers
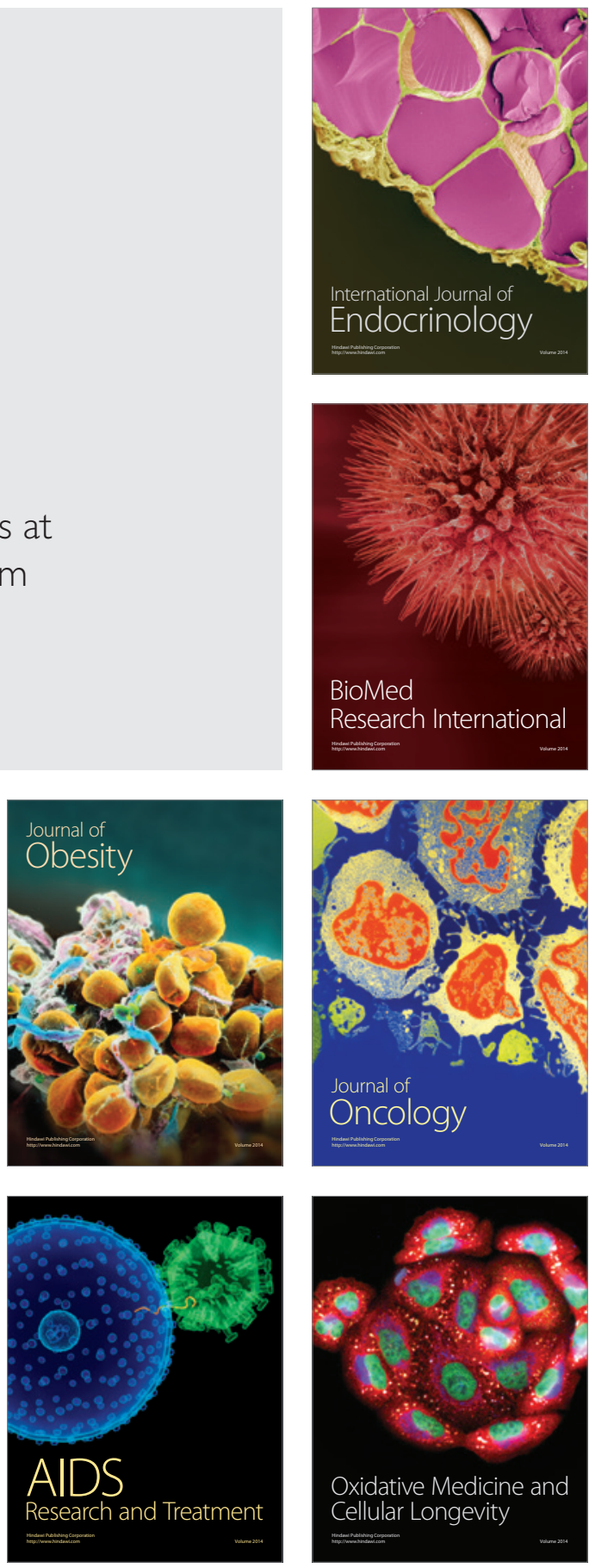